\title{
Resultados del lavado peritoneal con solución electrolizada en pacientes con abdomen agudo abordados por cirugía laparoscópica comparados con lavados con solución salina fisiológica o sin lavados
}

\author{
Results of abdominal cavity lavages with neutral electrolyzed water \\ in patients with acute abdomen approached by laparoscopic surgery \\ compared to physiological saline solution lavages or no lavages
}

Jorge Oliver Valdés Pineda, ${ }^{*}$ Luis Roberto Gutiérrez Hernández, ${ }^{*}$ Jazmín López Cabrera, ${ }^{*}$ Gabriela Ruiz Mar, ${ }^{*}$ Andrea de la Rosa Bandini, ${ }^{*}$ Arturo Benítez Trejo, ${ }^{*, \dagger}$ Nicolás Mervitch Sigal, ${ }^{\ddagger}$ Ariana Cabrera Licona ${ }^{\S}$

\footnotetext{
* Servicio de Cirugía General del Hospital Nacional Homeopático de la Ciudad de México, México.

‡ Dirección Médica, Esteripharma México S.A. de C.V., Cuidad de México, México.

$\S$ Dirección de Investigación, Esteripharma S.A. de C.V., Atlacomulco, Estado de México, México.
}

RESUMEN

Introducción: Las soluciones electrolizadas de superoxidación con $\mathrm{pH}$ neutro (SES) son reconocidos antisépticos por ofrecer amplio espectro microbicida sin dañar mucosas o tejidos. Este trabajo exploró si su uso para el lavado peritoneal, durante procedimientos laparoscópicos de enfermos que sufren abdomen agudo quirúrgico, ingresados en el Hospital Nacional Homeopático de la Ciudad de México, ofrece ventajas sobre el lavado con solución salina fisiológica o sobre intervenir sin lavar. Material y métodos: Se realizó un estudio retrospectivo de cohorte, donde se compararon el desarrollo de complicaciones posquirúrgicas y los días de estancia hospitalaria entre los grupos

\section{ABSTRACT}

Introduction: Neutral electrolyzed water (SES) is a well-recognized broad-spectrum antiseptic that does not cause tissue damage. This work explored whether peritoneal lavages with neutral electrolyzed water during laparoscopic procedures, in patients with acute surgical abdomen admitted to the National Homeopathic Hospital in Mexico City, offers advantages over lavages with normal saline solution, or over intervening without lavages. Material and methods: A retrospective cohort study was carried out where development of postoperative complications and length of hospital stay were compared between groups without peritoneal lavages, lavages with normal saline solution, and those with

\section{Recibido: 07/12/2020. Aceptado: 02/02/2021.}

Correspondencia: Dra. Ariana Cabrera Licona

Dirección de Investigación, Esteripharma S.A de C.V. Libramiento Jorge Jiménez Cantú 412,

Col. Dos de abril, 50450, Atlacomulco, Estado de México, México. Tel. 5550613500

E-mail: acabrera@esteripharma.com.mx

Citar como: Valdés PJO, Gutiérrez HLR, López CJ, Ruiz MG, de la Rosa BA, Benítez TA, et al. Resultados del lavado peritoneal con solución electrolizada en pacientes con abdomen agudo abordados por cirugía laparoscópica comparados con lavados con solución salina fisiológica o sin lavados. Rev Mex Cir Endoscop. 2020; 21 (4): 191-199. https://dx.doi.org/10.35366/101218 
sin lavados del peritoneo, lavados con solución fisiológica salina, o bien usando SES al $0.002 \%$ de especies activas de cloro y oxígeno. Los datos se analizaron utilizando pruebas de ANOVA de dos colas y razón de momios con $p<0.05$. Resultados: Ningún paciente lavado con SES desarrolló trastornos posquirúrgicos, y esto fue significativo contrastado con no lavar [OR $=0.02343$ (IC95\% 0.001286-0.4268)], o contra lavar recurriendo a solución fisiológica salina [OR $=0.05051$ (IC95\% 0.002632-0.9690)]. El grupo lavado con SES mostró mayor significancia en la disminución de los días de estadía hospitalaria (1.61 $\pm 0.76)$ que el grupo lavado con solución fisiológica salina $(2.40 \pm 1.14)$ o que el grupo sin lavados $(2.36 \pm 1.46)$. Conclusiones: Se encontró asociación positiva entre realizar dichas limpiezas peritoneales durante la cirugía laparoscópica y menor incidencia de complicaciones posquirúrgicas y de tiempo de permanencia hospitalaria, que fue más significativa usando la solución electrolizada que al emplear la salina fisiológica. No realizar lavados se asoció con mayor número de pacientes con complicaciones tras la cirugía y tiempos más largos de estancia hospitalaria.

Palabras clave: Solución electrolizada de superoxidación, lavado peritoneal, laparoscopía, abdomen agudo quirúrgico, complicaciones posquirúrgicas. electrolyzed water, with $0.002 \%$ of active chlorine and oxygen species. Data were analyzed with two-tailed ANOVA and odds ratio tests. Results: No patient lavaged with SES developed postsurgical difficulties, and this was significant with respect to not lavage [OR $=0.02343(95 \%$ $\mathrm{Cl}$ 0.001286-0.4268)] or lavage with physiological saline solution [OR $=0.05051(95 \% \mathrm{Cl} 0.002632-0.9690)]$. The group lavaged with SES showed greater significance in the reduction of days of hospital stay (1.61 $\pm 0.76)$ than the group lavaged with physiological saline solution $(2.40 \pm$ $1.14)$ or the group without lavages $(2.36 \pm 1.46)$. Conclusions: $A$ positive association was found between peritoneal lavages during laparoscopic surgery and a lower incidence of both postoperative complications and length of hospital stay, which was more significant with electrolyzed water than with physiological solution, while no lavages was associated with a greater number of patients with postoperative complications and longer hospital stay.

Keywords: Neutral electrolyzed water, peritoneal lavages, laparoscopy, acute surgical abdomen, postoperative complications.

\section{INTRODUCCIÓN}

El abdomen agudo es un síndrome clínico de dolor abdominal con evolución menor a 48 horas o hasta seis días. Son causa común de abdomen agudo la apendicitis aguda, colecistitis complicadas, úlceras pépticas perforadas y diverticulitis aguda, que de agravarse pueden generar peritonitis e incluso sepsis abdominal. ${ }^{1}$ Clínicamente, en los pacientes ingresados con dolor muy acentuado de abdomen, a fin de estimar el riesgo de desarrollar sepsis abdominal se pueden utilizar los criterios del síndrome de respuesta inmune sistémica (SRIS); ${ }^{2}$ ante diagnóstico presuntivo, las Guías Internacionales para el Manejo de Sepsis y Choque Séptico sugieren realizar control de la causa primaria usando la técnica quirúrgica menos invasiva (preferentemente cirugía laparoscópica), junto con el aseo de la cavidad abdominal y la profilaxis antibiótica, buscando evitar trastornos severos. ${ }^{3}$ En la práctica, la solución salina fisiológica y diluciones de antibióticos se utilizan como fluidos para el lavado abdominal, aunque su uso es controversial y puede tener efectos adversos, por ejemplo toxicidad al tejido e inducción de resistencia bacteriana. ${ }^{4,5}$

En cambio, las soluciones electrolizadas de superoxidación con pH neutro (SES) son antisépticos de amplio espectro que no causan resistencia bacteriana ni citotoxicidad y han sido utilizadas exitosamente como fluidos de lavado en laparotomía. ${ }^{6-9}$ Sus potenciales ventajas como soluciones de lavado durante procedimientos laparoscópicos de abdomen agudo mostrando riesgo de sepsis abdominal no se han establecido. El objetivo de este trabajo fue comparar la evolución de pacientes del Hospital Nacional Homeopático de la Ciudad de México con abdomen agudo quirúrgico de diversas etiologías, y que fueron abordados por laparoscopía. En los pacientes considerados se realizaron lavados de la cavidad peritoneal ya sea con mezcla electrolizada de superoxidación de $\mathrm{pH}$ neutro al $0.002 \%$ de especies activas de cloro y oxígeno, o usando solución fisiológica, o bien no se efectuaron lavados en tanto se desarrollaba el proceso. Específicamente, se compararon la incidencia de complicaciones posquirúrgicas y los tiempos de estancia hospitalaria. En general, se encontró asociación positiva entre lavar la cavidad abdominal durante la cirugía laparoscópica y menor incidencia de dificultades posquirúrgicas y de tiempo de estadía hospitalaria, la cual fue más significativa utilizando la preparación electrolizada que aplicando la solución fisiológica. No realizar ningún lavado se asoció con mayor número de enfermos con alguna complicación posquirúrgica y tiempos más largos de permanencia en el hospital.

\section{MATERIAL Y MÉTODOS}

Se llevó a cabo un estudio retrospectivo de cohorte, analizando expedientes de enfermos con diagnóstico confirmado de abdomen agudo quirúrgico por etiologías diversas, y que tuvieron abordaje quirúrgico laparoscópico en la Unidad de Cirugía General del Hospital Nacional Homeopático, en el periodo comprendido desde julio de 2016 hasta junio de 2017.

De un total de 350 expedientes se seleccionaron los que cumplieron con los criterios de inclusión: hombres 
o mujeres, mayores de edad, tener mínimo dos criterios clínicos de SRIS positivos [leucocitosis ( $>12,000$ células/ $\mathrm{mL}$ ) o leucopenia ( $>12,000$ células $/ \mathrm{mL}$ ), taquicardia (> 90 latidos por minuto), taquipnea ( $>20$ respiraciones por minuto), fiebre $\left(>38^{\circ} \mathrm{C}\right)$ o hipotermia $\left(<35.5^{\circ} \mathrm{C}\right)$, hipotensión ( $\leq 100 \mathrm{mmHg})$ ], que durante la operación la cavidad peritoneal hubiera sido lavada con solución electrolizada, mezcla fisiológica o que no hubieran recibido lavados y con antibioterapia similar pre- y posoperatoria.

Los criterios de exclusión fueron: no contar con alguno de los valores de ingreso de signos vitales antes mencionados, presentar inmunodeficiencias, comorbilidades, cáncer u otras patologías no asociadas a la etiología del abdomen agudo quirúrgico, haber recibido otro tipo de antibioterapia después de la cirugía.

En total, 78 expedientes cumplieron con los criterios de inclusión. En 25 pacientes no se practicaron lavados (Gpo$\mathrm{SL})$. En 22 se registró el uso de solución salina fisiológica (Gpo-SSF) y en 31 se utilizó preparación electrolizada de superoxidación de $\mathrm{pH}$ neutro (Gpo-SES).

Las profilaxis se realizaron conectando al irrigador bolsas de SSF o de SES (Estericide ${ }^{\circledR}$ Antiséptico Irrigación Quirúrgica, al $0.002 \%$ de especies activas de cloro y oxígeno con potencial REDOX $750-950 \mathrm{mV}$, registro sanitario 0820C2016 SSA). Se aseguró la irrigación pélvica y entre asas en cuatro cuadrantes hasta que no se observó líquido purulento en la cavidad peritoneal. En el Gpo-SSF se utilizaron volúmenes en el rango de $100 \mathrm{~mL}$ a dos litros, mientras que en el Gpo-SES desde $60 \mathrm{~mL}$ hasta un litro. En el Gpo-SL, no se conectó ningún líquido antiséptico al irrigador y se limpió el pus abdominal sólo aplicando succión. A todos los pacientes se les administró, antes de la cirugía, ceftriaxona ( 2 g) más metronidazol (500 $\mathrm{mg}$ ) intravenoso, y dos dosis más en un lapso de 48 horas después de ésta.

La tasa de complicaciones posquirúrgicas, principalmente de abscesos intraabdominales e infecciones del puerto quirúrgico, se consideraron las variables de resultado primarias. La medida secundaria de resultados incluyó los cambios en los valores de presión arterial, frecuencia cardiaca, frecuencia respiratoria, temperatura, cuenta leucocitaria y duración de la estancia hospitalaria. Las diferencias intergrupales que acoplan los criterios de diagnóstico clínico de inflamación sistémica y los días de permanencia hospitalaria se analizaron con las pruebas estadísticas ANOVA de dos colas y Tukey. La relación que conecta los lavados al desarrollo de dificultades posquirúrgicas se estableció calculando los valores de razón de probabilidad-OR (odds ratio), aplicando la prueba de $\chi^{2}$ exacta de Fisher. ${ }^{10}$ Se consideraron significativos cambios con $\mathrm{p}<0.05 .{ }^{11}$ La realización del estudio recibió la aprobación del comité de ética del hospital.

\section{RESULTADOS}

Entre las características basales de los grupos no hubo diferencia significativa. La media de años de edad fue de $35.8 \pm 13.26$ en el conjunto donde no se realizaron lavados de la cavidad peritoneal (Gpo-SL), fue de $41.5 \pm 20.03$ en el grupo donde se efectuaron lavados con SSF (Gpo-SSF) y de $35.9 \pm 14.12$ en el otro donde se utilizó SES (Gpo$\mathrm{SES})$; entre ellos no hubo diferencia estadística significativa, $\mathrm{p}=0.3680$ (Tabla 1). En cuanto al sexo, tampoco hubo diferencia significativa, con valores de $\mathrm{p}$ mayores a 0.05 en cada comparación: Gpo-SL versus Gpo-SSF, Gpo-SL versus Gpo-SES y Gpo-SSF versus Gpo-SES (Tabla 1). Sin embargo, hubo un porcentaje mayor de hombres en los tres, principalmente en el Gpo-SES con $77.4 \%$.

Por otra parte, se estableció que un mínimo de dos signos clínicos del síndrome de respuesta inmune sistémica (SRIS) positivos indicaban un posible riesgo de sepsis abdominal, por lo que se analizó si hubo desigualdades significativas que diferenciaran la incidencia de leucocitosis, taquicardia, taquipnea, hipotensión, fiebre o hipotermia entre los grupos, previo a la intervención quirúrgica (Tabla 1). No se encontraron diferencias estadísticamente significativas en la incidencia de estos signos clínicos (Tabla 1). Cabe señalar que la leucocitosis se presentó en la mayoría de los enfermos en los tres conjuntos (Tabla 1), seguida de taquicardia y taquipnea, mientras que un muy reducido número de pacientes presentó fiebre o hipotermia (Tabla 1).

Las patologías causantes del abdomen agudo fueron: apendicitis aguda no complicada, en el Gpo-SL con 48\% de los casos, 31.82\% en el Gpo-SSF y 25.8\% en el Gpo-SES, revelando una diferencia significativa entre los dos primeros con $p=0.0326$ (Tabla 1). La segunda patología con mayor incidencia fue la apendicitis aguda complicada, con 24\% en Gpo-SL, 36.37\% en Gpo-SSF y $41.94 \%$ en Gpo-SES. En este caso hubo diferencia significativa entre Gpo-SL y Gpo-SES con $p=0.0024$, y entre Gpo-SSF y Gpo-SES, con $p=0.0326$ (Tabla 1). La tercera patología que manifestó incidencia elevada fue la colecistitis crónica litiásica aguda con piocolecisto, dando cifras de $8,9.1$ y $12.9 \%$, pero $\sin$ diferencia significativa (Tabla 1).

Se analizaron los cambios en los signos clínicos posoperatorios, el desarrollo de trastornos posquirúrgicos y los días de estancia hospitalaria. La leucocitosis permaneció en seis enfermos del Gpo-SL, cuatro del Gpo-SSF y en seis del Gpo-SES; debe señalarse que varios pacientes pidieron el alta voluntaria y no se realizó esta determinación en ellos. Al analizar el conteo de leucocitos se observó disminución estadísticamente significativa únicamente en los que se realizó lavado, Gpo-SES ( $p=0.0004)$ y GpoSSF $(p=0.0398)$ versus el grupo sin lavados (Figura 1). 
Tabla 1: Características generales, criterios clínicos y etiologías del abdomen agudo en los pacientes de los grupos.

\begin{tabular}{|c|c|c|c|c|}
\hline Características & Gpo-SL (N = 25) & Gpo-SSF (N = 22) & Gpo-SES (N = 31) & p \\
\hline $\begin{array}{l}\text { Edad, media } \pm \mathrm{DE} \\
\text { Género, } \mathbf{n}(\%)\end{array}$ & $35.83 \pm 13.26$ & $41.50 \pm 20.03$ & $35.90 \pm 14.12$ & 0.3680 \\
\hline Masculino & $16(64.0)$ & $13(59.1)$ & $24(77.4)$ & $\begin{array}{l}0.9033^{a} \\
0.5685^{b} \\
0.4109^{c}\end{array}$ \\
\hline Femenino & $9(36.0)$ & $9(40.9)$ & $7(22.6)$ & $\begin{array}{r}>0.9999^{a} \\
0.9546^{b} \\
0.9546^{c}\end{array}$ \\
\hline \multicolumn{5}{|l|}{ Signos clínicos al ingreso } \\
\hline Leucocitosis & 18 & 17 & 25 & $\begin{array}{l}0.9521^{a} \\
0.1409^{b} \\
0.0880^{c}\end{array}$ \\
\hline Taquicardia & 12 & 9 & 12 & $\begin{array}{r}0.6541^{a} \\
>0.9999^{b} \\
0.6541^{c}\end{array}$ \\
\hline Taquipnea & 11 & 12 & 9 & $\begin{array}{l}0.9521^{a} \\
0.8242^{b} \\
0.6541^{c}\end{array}$ \\
\hline Hipotensión & 6 & 8 & 5 & $\begin{array}{l}0.8242^{a} \\
0.9521^{b} \\
0.6541^{c}\end{array}$ \\
\hline Fiebre & 1 & 3 & 1 & $\begin{array}{r}0.8242^{a} \\
>0.9999^{b} \\
0.8242^{c}\end{array}$ \\
\hline Hipotermia & 1 & 1 & 0 & $\begin{array}{r}>0.9999^{a} \\
0.9521^{b} \\
0.9521^{c}\end{array}$ \\
\hline \multicolumn{5}{|c|}{ Etiología del abdomen agudo quirúrgico } \\
\hline $\begin{array}{l}\text { Apendicitis aguda no } \\
\text { complicada (AANC) }\end{array}$ & 12 & 7 & 8 & $\begin{array}{l}0.0326^{\mathrm{a}, \star} \\
0.1011^{\mathrm{b}} \\
0.8556^{\mathrm{c}}\end{array}$ \\
\hline AANC + colecistitis aguda & 0 & 1 & 0 & $\begin{array}{r}0.8556^{a} \\
>0.9999^{b} \\
0.8556^{c}\end{array}$ \\
\hline $\begin{array}{l}\text { Apendicitis aguda } \\
\text { complicada (AAC) }\end{array}$ & 6 & 8 & 13 & $\begin{array}{l}0.5420^{\mathrm{a}} \\
0.0024^{\mathrm{b}, \star *} \\
0.0326^{\mathrm{c}, *}\end{array}$ \\
\hline$A A C+$ peritonitis generalizada & 1 & 1 & 1 & $\begin{array}{l}>0.9999^{a} \\
>0.9999^{b} \\
>0.9999^{c}\end{array}$ \\
\hline $\mathrm{AAC}+$ piocolecisto & 0 & 0 & 1 & $\begin{array}{r}0.9999^{a} \\
0.8556^{b} \\
0.8556^{c}\end{array}$ \\
\hline AAC + salpingitis bilateral reactiva & 1 & 0 & 0 & $\begin{array}{r}0.8556^{a} \\
0.8556^{b} \\
>0.9999^{c}\end{array}$ \\
\hline $\begin{array}{l}\text { Colecistitis crónica litiásica } \\
\text { aguda }(C C L A)+\text { piocolecisto }\end{array}$ & 2 & 2 & 4 & $\begin{array}{r}0.9999^{a} \\
0.5420^{b} \\
0.5420^{c}\end{array}$ \\
\hline
\end{tabular}


Continúa la Tabla 1: Características generales, criterios clínicos y etiologías del abdomen agudo en los pacientes de los grupos.

\begin{tabular}{|c|c|c|c|c|}
\hline Características & Gpo-SL $(\mathrm{N}=25)$ & Gpo-SSF $(N=22)$ & Gpo-SES (N = 31) & $\mathrm{p}$ \\
\hline \multicolumn{5}{|l|}{ Etiología del abdomen agudo quirúrgico } \\
\hline $\begin{array}{l}\text { Colecistitis }+ \text { piocolecisto }+ \\
\text { absceso perivesicular }\end{array}$ & 0 & 0 & 1 & $\begin{array}{r}>0.9999^{a} \\
0.8556^{b} \\
0.8556^{c}\end{array}$ \\
\hline $\begin{array}{l}\text { Colecistitis + piocolecisto + adherencias } \\
\text { epiplón y hernia supraumbilical }\end{array}$ & 0 & 0 & 1 & $\begin{array}{r}>0.9999^{a} \\
0.8556^{b} \\
0.8556^{\circ}\end{array}$ \\
\hline $\begin{array}{l}\text { Colecistitis + piohidrocolecisto } \\
+ \text { adherencias }\end{array}$ & 0 & 0 & 1 & $\begin{array}{r}0.9999^{a} \\
0.8556^{b} \\
0.8556^{\circ}\end{array}$ \\
\hline $\begin{array}{l}\text { Colecistitis + piocolecisto } \\
\text { perforado sellado }\end{array}$ & 2 & 0 & 0 & $\begin{array}{r}0.5420^{a} \\
0.5420^{b} \\
0.9999^{\circ}\end{array}$ \\
\hline $\begin{array}{l}\text { Colecistitis + piocolecisto + } \\
\text { síndrome de Mirizzi tipo I }\end{array}$ & 1 & 0 & 1 & $\begin{array}{r}0.8556^{a} \\
>0.9999^{b} \\
0.8556^{\circ}\end{array}$ \\
\hline $\begin{array}{l}\text { Enfermedad diverticular } \\
\text { Hinchey IIA }\end{array}$ & 0 & 1 & 0 & $\begin{array}{r}0.8556^{a} \\
>0.9999^{b} \\
0.8556^{\circ}\end{array}$ \\
\hline Enfermedad diverticular Hinchey III & 0 & 1 & 0 & $\begin{array}{r}0.8556^{a} \\
>0.9999^{b} \\
0.8556^{\circ}\end{array}$ \\
\hline Piosalpinx & 0 & 1 & 0 & $\begin{array}{r}0.8556^{a} \\
>0.9999^{b} \\
0.8556^{\circ}\end{array}$ \\
\hline
\end{tabular}

Prueba de comparación múltiple de Tukey donde: ${ }^{a}$ Gpo-SL vs Gpo-SSF, ${ }^{b}$ Gpo-SL vs Gpo-SES, ${ }^{\circ}$ Gpo-SSF vs Gpo-SES; * $p<0.05,{ }^{* *} p<0.01$.

Así mismo, dos pacientes del Gpo-SL tuvieron taquipnea y tres en el Gpo-SSF y en el Gpo-SES, sin diferencia estadística entre ellos (Tabla 2). Con respecto a la hipotensión, un paciente en el Gpo-SSF y tres en el Gpo-SES mantuvieron la tensión baja, siendo significativa la diferencia ente el Gpo-SL y el Gpo-SES con p = 0.0482 (Tabla 2). Ninguno de los pacientes en los tres grupos presento fiebre o hipotermia.

El análisis de la incidencia de complicaciones posoperatorias en cada grupo mostró que las principales fueron los abscesos y la infección de la herida quirúrgica (Tabla 2). Se encontró diferencia estadísticamente significativa entre el número de abscesos en el Gpo-SSF y el Gpo-SES con $p=0.0251$. También fue significativamente distinta la incidencia de dificultades quirúrgicas en el Gpo-SL en comparación con el Gpo-SES con p = 0.0251 (Tabla 2). En general, el Gpo-SL presentó el mayor número de enfermos con complicaciones: 10 , equivalentes a $40 \%$ de la población. En el Gpo-SSF, cinco enfermos (22.73\%) presentó alguna complicación y, en contraste, ningún paciente del
Gpo-SES. El análisis de razón de probabilidades reveló que no hubo diferencia entre no realizar lavados o lavar con SSF y el desarrollo de complicaciones posquirúrgicas $[\mathrm{OR}=0.4412$ (IC95\% 0.1228-1.585)], mientras que sí existió una asociación positiva entre lavar usando SES y no desarrollar complicaciones, en comparación con el uso de SSF $[O R=0.05051$ (IC95\% 0.002632-0.9690)], y aún más significativa entre lavar utilizando SES y no realizar ninguno $[\mathrm{OR}=0.02343(\mathrm{IC} 95 \% 0.001286$ 0.4268)] (Tabla 3).

Finalmente, respecto al tiempo de permanencia hospitalaria, contado desde el ingreso a cirugía hasta el alta de los pacientes, hubo diferencia significativa entre el Gpo-SES y el Gpo-SSF ( $p=0.0366)$, con un promedio de días de estadía de $1.613 \pm 0.7606$ y $2.409 \pm 1.141$, respectivamente. También hubo diferencia significativa del Gpo-SES versus Gpo-SL ( $p=0.0433$ ) con una estancia promedio en este último grupo de $2.360 \pm 1.469$ días. No hubo diferencia estadística entre Gpo-SSF y Gpo-SL (Figura 2). 


\section{DISCUSIÓN}

En este análisis retrospectivo se compararon los signos clínicos posoperatorios, las complicaciones posquirúrgicas y el tiempo de estancia hospitalario, en pacientes que presentaban abdomen agudo y que fueron atendidos mediante cirugías laparoscópicas. En dichas intervenciones se aplicaron lavados de la cavidad peritoneal con dilución fisiológica salina, con solución electrolizada de superoxidación de $\mathrm{pH}$ neutro al $0.002 \%$ de especies activas de cloro y oxígeno, o no se realizaron tales asepsias durante la operación. Sin embargo, se debe señalar que existe una serie de sesgos que hacen compleja la comparación, pues los grupos fueron pequeños y la población heterogénea.

En primera instancia, el grado de severidad de las patologías productoras del abdomen agudo fue distinto entre los pacientes de un mismo grupo y entre los grupos. A pesar de que se trató de uniformarlos con base en los criterios clínicos del síndrome de respuesta inmune sistémica como medida del riesgo de peritonitis y sepsis abdominal no fue posible evitar dicho sesgo. Por tanto, no contar con casos que tengan las mismas patologías y en mismo grado de severidad, y con una muestra mayor, son limitaciones de este trabajo.

Fue evidente, además, la parcialidad que implicó la decisión del cirujano de realizar o no lavados. Es percep-

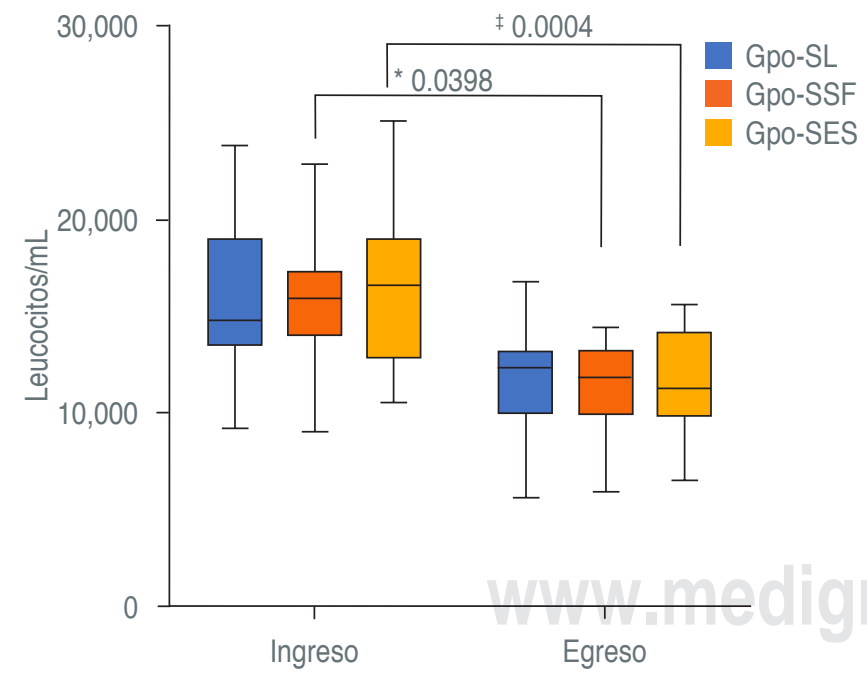

Figura 1: Comparación del cambio en el conteo de leucocitos, al ingreso y egreso de la cirugía laparoscópica entre los grupos Gpo-SL, GpoSSF y Gpo-SES. Datos analizados por comparación múltiple.

* Significancia estadística entre grupos con $p<0.05$.

₹ Significancia estadística entre grupos con $p<0.001$. No se encontró diferencia significativa en el grupo sin lavado $(p=0.1753)$. Se reporta el valor de desviación estándar. tible que los facultativos no realizaron estas limpiezas en aquellas patologías que no parecían acarrear riesgos de peritonitis, como la apendicitis aguda no complicada, que representó 40\% de los casos donde no se realizó lavado. En cambio, se realizaron lavados con SES en $41.94 \%$ de los casos de la variante complicada y usando SSF en 36.4\%, lo cual fue estadísticamente significativo.

Cabe destacar que, aunque en el grupo SES parece que se agrupan patologías con mayor riesgo de peritonitis, y el cirujano decidió lavar la cavidad, no se reportó ninguna dificultad posoperatoria y los enfermos fueron dados de alta en tiempos más cortos con respecto a los otros dos grupos analizados. En comparación, en el grupo sin lavado, donde aparentemente había casos con menor riesgo de peritonitis o sepsis, 40\% desarrolló alguna complicación posoperatoria, desde abscesos hasta un caso de fascitis necrosante, principalmente en los casos de inflamación aguda de apéndice tanto complicada como no complicada.

Los lavados aplicando SSF parecen disminuir el riesgo de trastornos después de la cirugía, en patologías que los cirujanos, al parecer, percibieron con mayor riego de sepsis, con $22.7 \%$ de incidencia, que en su mayoría fueron abscesos. Por consecuencia, la comparación entre estos conjuntos permite sugerir que lavar con SES podría disminuir la incidencia de desórdenes posoperatorios.

Para reafirmar estos resultados, se necesitan estudios prospectivos aleatorizados, con cohortes con la misma patología y severidad, que analicen los cambios en la carga bacteriana, que involucren otros parámetros laboratoriales de la respuesta inmune y en los que los pacientes reciban el mismo tratamiento antibiótico pre- y posoperatorio.

A pesar de que, como ya se mencionó, se precisan más estudios, estos resultados preliminares muestran que, mientras se aplican intervenciones laparoscópicas, los lavados de la cavidad del peritoneo empleando solución electrolizada de superoxidación pueden ser ventajosos frente a no lavar o lavar con SSF. Estos efectos pueden deberse a que la SES, además de ofrecer un efecto mecánico que por arrastre retira la materia orgánica de desecho tiene un efecto antiséptico de amplio espectro que, sin dañar tejidos y/o mucosas, lograría controlar la contaminación bacteriana mediante la eliminación de los gérmenes presentes en el lecho quirúrgico que pudieron quedar adheridos a las células mesoteliales peritoneales. Así mismo se ha reportado que las especies activas de cloro y oxígeno presentes en las SES pueden actuar a modo de segundos mensajeros en la quimiotaxis de células fagocíticas y en el control de la respuesta proinflamatoria. ${ }^{12}$

En conjunto, los lavados valiéndose de SES evitarían el desarrollo de focos de infección, en contraste con aquellos con SSF, de los cuales se ha reportado que pueden no ser 
efectivos en el intento de eliminar las bacterias adheridas y pueden causar abscesos posquirúrgicos lejanos, además de que pueden diluir los mediadores de la fagocitosis. ${ }^{13}$ Los resultados de este estudio retrospectivo son coherentes con las investigaciones en las que se utilizó SES para lavado de cavidad peritoneal durante laparotomías, donde se reportó

\section{Tabla 2: Cambios en los criterios clínicos al egreso y complicaciones posquirúrgicas en los grupos.}

\begin{tabular}{|c|c|c|c|c|}
\hline Signos clínicos al egreso & Gpo-SL (N = 25) & Gpo-SSF (N = 22) & Gpo-SES (N = 31) & $\mathrm{p}$ \\
\hline Leucocitosis & 6 & 4 & 6 & $\begin{array}{r}0.2055^{a} \\
>0.9999^{b} \\
0.2055^{c}\end{array}$ \\
\hline Taquicardia & 0 & 0 & 1 & $\begin{array}{r}>0.9999^{a} \\
0.6398^{b} \\
0.6398^{c}\end{array}$ \\
\hline Taquipnea & 2 & 3 & 3 & $\begin{array}{r}0.6398^{a} \\
0.6398^{b} \\
>0.9999^{c}\end{array}$ \\
\hline Hipotensión & 0 & 1 & 3 & $\begin{array}{l}0.6398^{a} \\
0.0482^{b, \star} \\
0.2055^{c}\end{array}$ \\
\hline Fiebre & 0 & 0 & 0 & $>0.9999$ \\
\hline Hipotermia & 0 & 0 & 0 & $>0.9999$ \\
\hline \multicolumn{5}{|c|}{ Complicaciones posquirúrgicas y etiología del abdomen agudo quirúrgico } \\
\hline Seroma & 0 & $\begin{array}{c}1 \\
{ }^{\circ} \mathrm{AAC}\end{array}$ & 0 & $\begin{array}{r}0.5924^{a} \\
>0.9999^{b} \\
0.5924^{c}\end{array}$ \\
\hline Absceso & $\begin{array}{c}2 \\
{ }^{\circ} \mathrm{CCLA}+\text { piocolecisto } \\
{ }^{\circ} \mathrm{AAC} \\
+ \text { peritonitis generalizada }\end{array}$ & $\begin{array}{c}3 \\
{ }^{\circ} \mathrm{CCLA}+\text { piocolecisto } \\
{ }^{\circ} \mathrm{AAC} \\
{ }^{\circ} \mathrm{AAC}+ \\
\text { peritonitis generalizada }\end{array}$ & 0 & $\begin{array}{l}0.5924^{a} \\
0.1517^{b} \\
0.0251^{c, *}\end{array}$ \\
\hline $\begin{array}{l}\text { Infección de la } \\
\text { herida quirúrgica }\end{array}$ & $\begin{array}{c}3 \\
{ }^{\circ} \mathrm{AANC} \\
{ }^{\circ} \mathrm{AAC} \\
{ }^{\circ} \mathrm{AAC}+\text { salpingitis bilateral reactiva }\end{array}$ & $\stackrel{1}{\circ}$ & 0 & $\begin{array}{l}0.1517^{a} \\
0.0251^{b, \star} \\
0.5924^{c}\end{array}$ \\
\hline $\begin{array}{l}\text { Absceso + infección de } \\
\text { la herida quirúrgica }\end{array}$ & CCLA + piocolecisto & 0 & 0 & $\begin{array}{r}0.5924^{a} \\
0.5924^{b} \\
>0.9999^{c}\end{array}$ \\
\hline $\begin{array}{l}\text { Distensión y dolor } \\
\text { periumbilical }\end{array}$ & $\begin{array}{c}1 \\
\text { AANC }\end{array}$ & 0 & 0 & $\begin{array}{r}0.5924^{a} \\
0.5924^{b} \\
>0.9999^{c}\end{array}$ \\
\hline $\begin{array}{l}\text { Colecciones intrabdominales + } \\
\text { fascitis necrosante + hematoma }\end{array}$ & $\begin{array}{c}1 \\
\text { AAC }\end{array}$ & 0 & 0 & $\begin{array}{r}0.5924^{a} \\
0.5924^{b} \\
>0.9999^{c}\end{array}$ \\
\hline Hidrocolecisto & $\begin{array}{c}1 \\
\text { AANC }\end{array}$ & 0 & 0 & $\begin{array}{r}0.5924^{\mathrm{a}} \\
0.5924^{\mathrm{b}} \\
>0.9999^{\mathrm{c}}\end{array}$ \\
\hline $\begin{array}{l}\text { Piocolecisto con } \\
\text { apendicitis reactiva }\end{array}$ & $\begin{array}{c}1 \\
\text { AANC }\end{array}$ & 0 & 0 & $\begin{array}{r}0.5924^{a} \\
0.5924^{b} \\
>0.9999^{c}\end{array}$ \\
\hline Total, $\mathrm{n}(\%)$ & $10(40.00)$ & $5(22.73)$ & 0 & \\
\hline
\end{tabular}

Prueba de comparación múltiple de Tukey donde: ${ }^{a}$ Gpo-SL vs Gpo-SSF, ${ }^{b}$ Gpo-SL vs Gpo-SES, ${ }^{\circ}$ Gpo-SSF vs Gpo-SES; * $p<0.05$.

$\mathrm{CCLA}=$ Colecistitis crónica litiásica aguda, $\mathrm{AAC}=$ Apendicitis aguda complicada, $\mathrm{AANC}=$ Apendicitis aguda no complicada . 
Tabla 3: Asociación entre Gpo-SL, Gpo-SSF y Gpo-SES y el desarrollo de complicaciones posoperatorias determinado por análisis de razón de probabilidades o razón de momios (OR [odds ratio]).

\begin{tabular}{lccc} 
Tratamiento & $p$ & OR & IC 95\% \\
\hline Gpo-SSF vs Gpo-SL & 0.2301 & 0.4412 & $0.1228-1.585$ \\
Gpo-SES vs Gpo-SL & $<0.0001^{*}$ & 0.02343 & $0.001286-0.4268$ \\
Gpo-SES vs Gpo-SSF & $0.0092^{* *}$ & 0.05051 & $0.002632-0.9690$ \\
\hline${ }^{*}$ Significancia estadística entre grupos con $p<0.0001$. & & \\
${ }^{* *}$ Significancia estadística entre grupos con $p<0.01$. & &
\end{tabular}

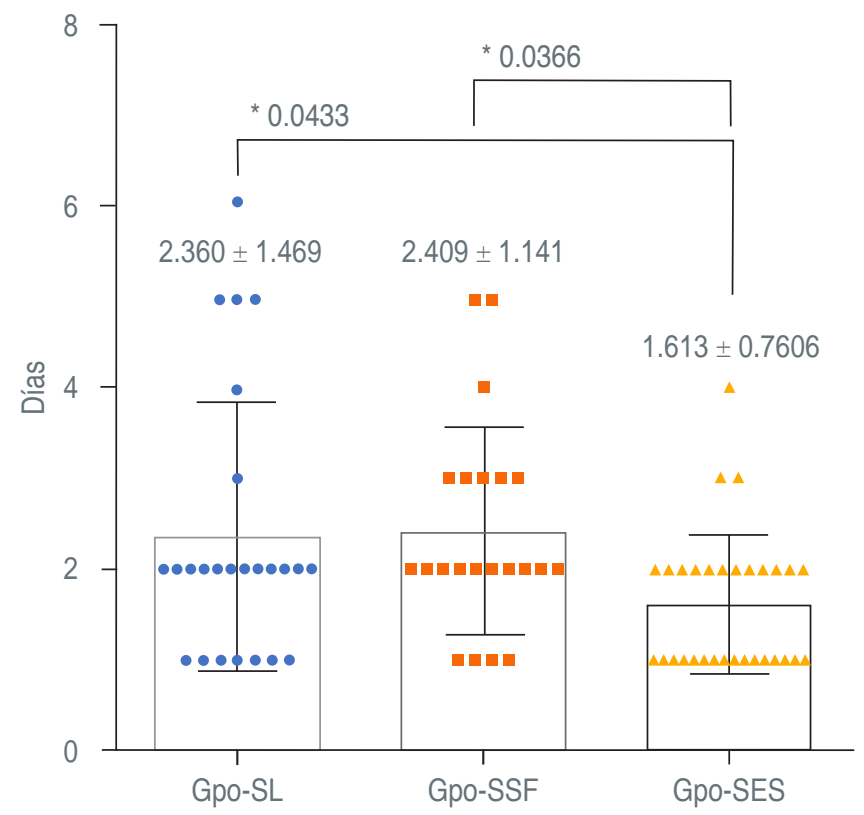

Figura 2: Comparación del tiempo de estancia hospitalaria entre los grupos Gpo-SL, Gpo-SSF y Gpo-SES. Datos del promedio de días de estancia analizados por comparación múltiple.

* Significancia estadística entre grupos con $p<0.05$. Se reporta el valor de desviación estándar.

disminución de la incidencia de infecciones, mas no ausencia. Aunque se debe señalar que en esos trabajos se utilizó la SES diluida con solución fisiológica salina. En el presente reporte los lavados se realizaron aplicando el fluido sin diluir ya que, a fin de asegurar la actividad antiséptica de amplio espectro y controlar de forma efectiva a cualquier tipo de microorganismo patógeno, se debe respetar la concentración de especies activas de cloro y oxígeno, el $\mathrm{pH}$ neutro (6.5-7.5) y el potencial REDOX (750-950 mV). ${ }^{14}$

\section{CONCLUSIÓN}

El análisis retrospectivo de los resultados de no lavar la cavidad peritoneal, lavar con solución salina fisiológica o bien usando la electrolizada de superoxidación, durante la cirugía laparoscópica de pacientes que padecen abdomen agudo por distintas etiologías, mostró que las complicaciones posoperatorias disminuyen, así como los días de estancia hospitalaria en los enfermos que recibieron tales lavados, siendo más significativos los resultados en aquellos cuya cavidad se lavó con SES. Estos resultados preliminares obtenidos en una muestra pequeña y heterogénea deben ser valorados en un estudio prospectivo controlado y aleatorizado en una población más grande para que sean confirmados.

\section{AGRADECIMIENTOS}

A la Dra. Brenda Paz-Michel por sus valiosos comentarios en la revisión del artículo.

\section{REFERENCIAS}

1. Gorordo-Delsol L, Pérez-Nieto OR, Porras-Escorcia O, Altamirano-Arcos CA. Sepsis abdominal: fisiopatología, diagnóstico y tratamiento. Rev Mex Cirugía del Apar Dig. 2015; 4: 110-117.

2. Zhang W, Zheng Y, Feng X, Chen M, Kang Y. Systemic inflammatory response syndrome in sepsis-3: A retrospective study. BMC Infect Dis. 2019; 19: 139.

3. Rhodes A, Evans LE, Alhazzani W, Levy MM, Antonelli $M$, Ferrer $R$ et al. Surviving sepsis campaign: international guidelines for management of sepsis and septic shock: 2016. Intensive Care Med. 2017; 43: 304-377.

4. Hajibandeh S, Hajibandeh S, Kelly A, Shah J, Khan RMA, Panda $\mathrm{N}$ et al. Irrigation versus suction alone in laparoscopic appendectomy: Is dilution the solution to pollution? A systematic review and meta-analysis. Surg Innov. 2018; 25: 174-182.

5. Edmiston CE, Leaper DJ. Intra-operative surgical irrigation of the surgical incision: what does the future hold - saline, antibiotic agents, or antiseptic agents? Surg Infect. 2016; 17: 656-564.

6. Singal R, Dhar S, Zaman M, Singh B, Sing V, Sethi S. Comparative evaluation of intra-operative peritoneal lavage with super oxidized solution and normal saline in peritonitis 
cases; randomized controlled trial. Maedica. 2016; 11: 277-285.

7. Khan S, Verma A, Shahid M, Khan M. Evaluation of preoperative peritoneal lavage by super-oxidized solution in peritonitis. Middle East J Intern Med. 2009; 2: 15-35.

8. Garg P, Garg A, Kumar A, Saini A, Sandhu A, Sharda V. Evaluation of intraoperative peritoneal lavage with superoxidized solution and normal saline in acute peritonitis. Arch Int Surg. 2013; 3: 43-48.

9. Nachón GFJ, Díaz TJ, Benítez OF, García GF, Santiago GJ, Martínez CAJ. Lavado peritoneal transoperatorio con solución electrolizada por selectividad iónica en peritonitis secundaria. Cir Gen. 2010; 32: 11-16.

10. Szumilas M. Explaining odds ratios. J Can Acad Child Adolesc Psychiatry. 2010; 19: 227-229.
11. GraphPad Prism versión 6.0 para Windows. GraphPad Software, La Jolla, California USA.

12. Holmstrom KM, Finkel T. Cellular mechanisms and physiological consequences of redox-dependent signalling. Nat Rev Mol Cell Biol. 2014; 15: 411-421.

13. Sun F, Wang H, Zhang F, Zhang X, Xing Z, Zhang S et al. Copious irrigation versus suction alone during laparoscopic appendectomy for complicated appendicitis in adults. J Investig Surg. 2018; 31: 342-346.

14. Kim HJ, Nkufi TC, Chelliah R, Oh DH. Sanitization Efficacy of Slightly Acidic Electrolyzed Water against pure cultures of Escherichia coli, Salmonella enterica, Typhimurium, Staphylococcus aureus and Bacillus cereus spores, in comparison with different water hardness. Sci Rep. 2019; 9: 1-14. 